\title{
Diagnostic accuracy of multidetector computed tomography scan in mediastinal masses assuming histopathological findings as gold standard
}

\author{
Somshankar Pandey, ${ }^{A, B E,}$, Usha Jaipal ${ }^{B, F}$, Nima Mannan', Rajkumar Yadav ${ }^{D}$ \\ SMS Medical College and Hospital, Jaipur, Rajasthan, India
}

\begin{abstract}
Purpose: Aim of the study was to: 1) present MDCT characteristics of different mediastinal mass lesions, 2) estimate proportion of benign and malignant mediastinal mass lesions based on MDCT findings, and 3) find out the diagnostic accuracy with sensitivity, specificity, positive predictive value, and negative predictive value of MDCT in mediastinal mass lesions assuming histopathology as gold standard.
\end{abstract}

Material and methods: This study was an analysis of 60 patients who underwent MDCT scan for characterisation of mediastinal mass lesion, and subsequently imaging findings were verified with pathological diagnosis.

Results: Out of 60 patients $65 \%$ were malignant and 35\% were benign. Metastatic carcinoma was the leading diagnosis. Sensitivity of MDCT in this study came out to be $94 \%$, specificity is $90 \%$, with a positive predictive value of $94 \%$ and negative predictive value of $90 \%$ with diagnostic accuracy of $93 \%$.

Conclusions: Mediastinal mass lesion can be accurately diagnosed with MDCT which is a non-invasive and easily available modality requiring clinical data for accurate diagnosis and management. Co-relation of MDCT findings with other imaging findings is complex and requires adequate clinical data for optimum diagnostic confidence.

Key words: MDCT, mediastinum, histopathology, ITMIG.

\section{Introduction}

Mediastinum is like a complex spider web. This is because of the presence of vital structures, e.g. heart and great vessels in the compact space between the two lungs. Mediastinal lesions are difficult to detect on X-ray because these lesions are of soft tissue densities and are surrounded by soft tissue structures. Previous studies have found conventional methods inadequate and patients frequently had to undergo invasive procedures for diagnosis. Here is where multidetector computed tomography (MDCT) can provide the answer. It provides information on the anatomical extent of the lesion and on the nature of the lesion (fat, fluid, calcification, etc.). MDCT can help to narrow the differential diagnosis or even reach the diagnosis without the need of further invasive procedures. The International Thymic Malignancy Interest Group (ITMIG) divided mediastinum into the following: prevascular, visceral, and paravertebral compartments (Figure 1) based on boundaries made by anatomical landmarks on CT [1], a detailed discussion of which is beyond the scope of the author.

\section{Material and methods}

\section{Study design}

A total of 60 patients participated in the study at our hospital for a period of two years, from November 2015 to

Correspondence address:

Dr. Somshankar Pandey, SMS Medical College and Hospital, C/0 Dr. Usha Jaipal Department of Radiodiagnosis, SMS Medical College, 302004 Jaipur, India,

phone: 919521153210, e-mail: somshankar.Jnv@gmail.com

Authors' contribution:

A Study design - B Data collection · C Statistical analysis · D Data interpretation - E Manuscript preparation · F Literature search · G Funds collection 


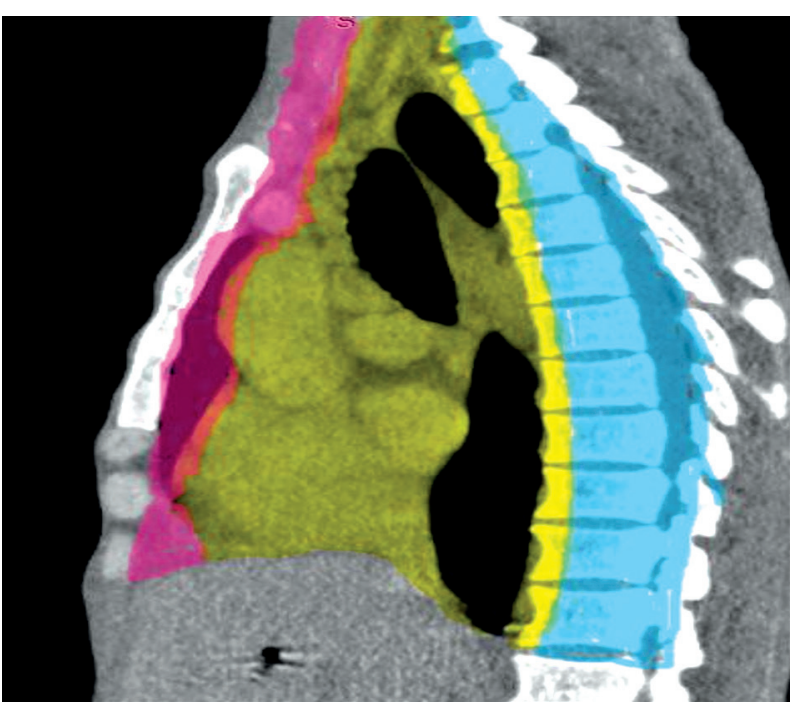

Figure 1. International Thymic Malignancy Interest Group (ITMIG) scheme. Pink area represents prevascular space, yellow area denotes visceral space, and line drawn $1 \mathrm{~cm}$ behind anterior border of vertebral body, i.e. area with sky blue colour, represents paravertebral space

November 2017. All relevant clinical information and details of laboratory investigation were recorded. All patients with clinical suspicion of mediastinal mass lesion or X-ray suggestion of mediastinal mass underwent thorough MDCT evaluation, and correlation with pathological findings was done in all cases.

Inclusion criteria were: 1) patients of both sexes of any age group clinically suspected to have mediastinal mass lesion; 2) suggestion of mediastinal widening/mass on chest radiograph; and 3) availability of histopathological examination report. Exclusion criteria were: 1) history of hypersensitivity to intravenous contrast agents; 2) eGFR

Table 1. Frequency and percentage of presenting complaints

\begin{tabular}{|l|c|c|}
\hline Complaints & No. & $\%$ \\
\hline Anorexia & 1 & 1.67 \\
\hline Asymptomatic & 1 & 1.67 \\
\hline Backache & 3 & 5.00 \\
\hline Chest pain & 9 & 15.00 \\
\hline Cough & 8 & 13.33 \\
\hline Dysphagia & 8 & 13.33 \\
\hline Dyspnoea & 13 & 21.67 \\
\hline Fatigue & 1 & 1.67 \\
\hline Fever & 9 & 15.00 \\
\hline Haemoptysis & 1 & 1.67 \\
\hline Paraesthesia & 1 & 1.67 \\
\hline Paraplegia & 1 & 1.67 \\
\hline Superior vena cava syndrome & 3 & 5.00 \\
\hline Weight loss & 1 & 1.67 \\
\hline Total & 60 & 100.00 \\
\hline
\end{tabular}
standard deviation while nominal variables were shown as a proportion (percentage). Chi-square test and Fisher Exact test were used for comparing proportions. $P$ values less than 0.05 were considered significant. Med Calc version 16.4 software was used for all statistical calculations.

\section{Results}

In this study, out of 60 cases, 37 cases $(61 \%)$ were males and 23 cases (38\%) were females. The most common age group to present with the mediastinal mass was between 41 and 50 years. In this study, dyspnoea was the most common clinical symptom, constituting $21.67 \%$, followed by fever at $15 \%$, chest pain at $15 \%$, while dysphagia contributed $13.33 \%$ (Table 1 ).

The most common compartment was prevascular space $-33 \%(n=20)$, followed by visceral space $-32.1 \%(n=19)$, paravertebral space constituted $-18.3 \%(n=11)$, and lesions involving multicompartment contributed $-16.6 \%$ $(n=10)$. 

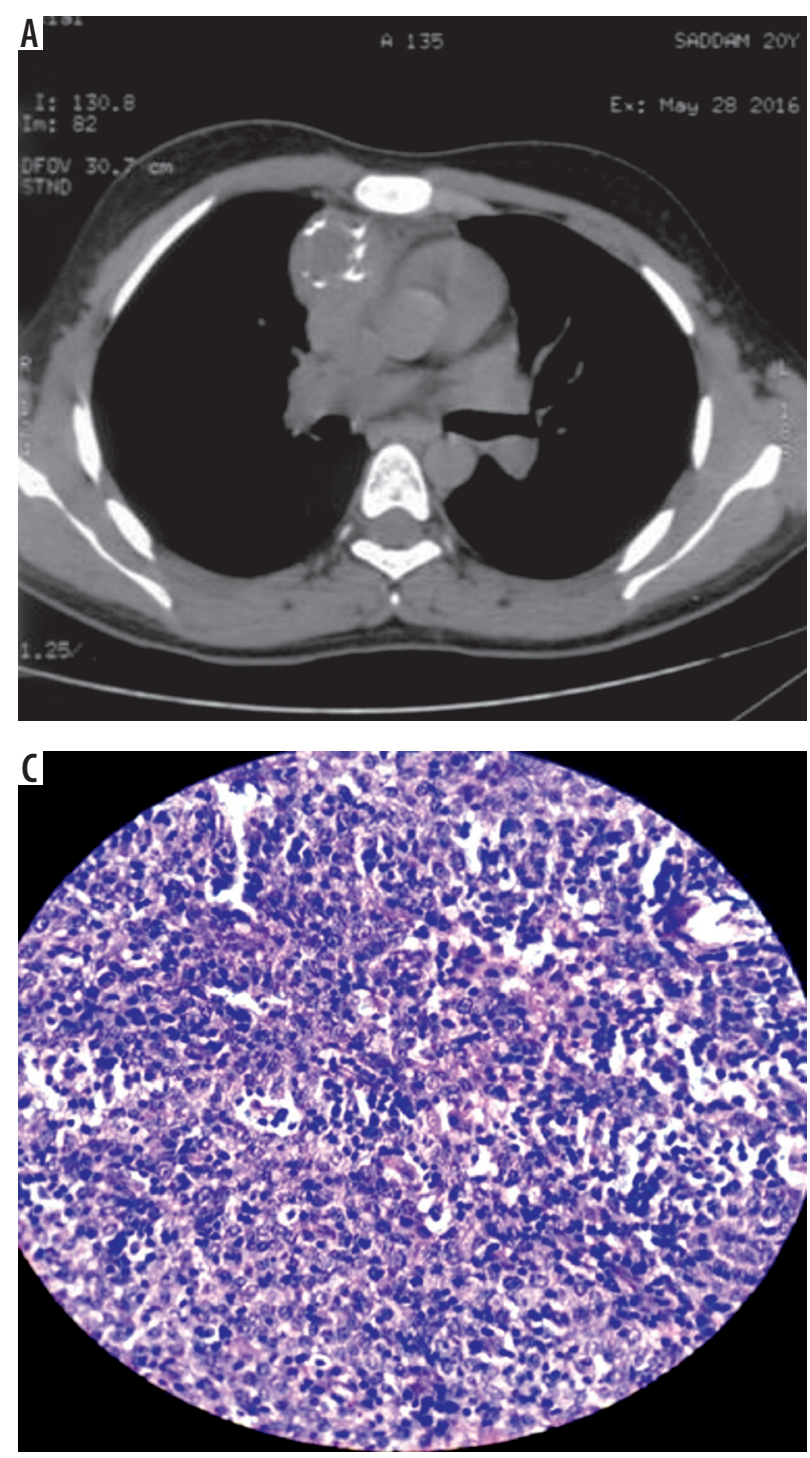

Among the prevascular space masses, thymoma (Figure 2) formed the majority, constituting $10 \%(n=6)$, followed by lymph nodal masses $8 \%(n=5)$. Among visceral compartment lymph nodal masses and oesophageal carcinoma were frequent, each $11 \%(n=7)$ among lymph nodal mass; most of them were metastatic in nature $8 \%(n=5)$. Of the posterior mediastinal masses, the majority contributed to neural tumours, constituting $10 \%$ $(n=6)$ followed by paravertebral abscess constituting 6\% $(n=4)$. In the study of 60 cases of neurogenic tumours, schwannoma (Figure 3 ) and neuroblastoma constituted 3\% each $(n=2)$, ganglioneuroblastoma $1.6 \%(n=1)$, and paraganglioma $1.6 \%(n=1)$ (Figure 4$)$. In the study, lymph nodal masses constituted $33 \%(n=20)$ of the total mediastinal mass. Among these the metastatic lymph node involvement was predominant, constituting $13 \%$ $(n=8)$, followed by lymphoma and tubercular lymph node enlargement $8 \%(n=5)$. In the study the majority of the masses showed heterogeneous attenuation, i.e. $46 \%$ ( $n=28)$ (Table 2) followed by homogenous attenuation; $26 \%(n=16)$, fatty $(n=1)$ fluid density, heterogenous enhancement $(n=25)$, homogenous enhancement $(n=17)$,

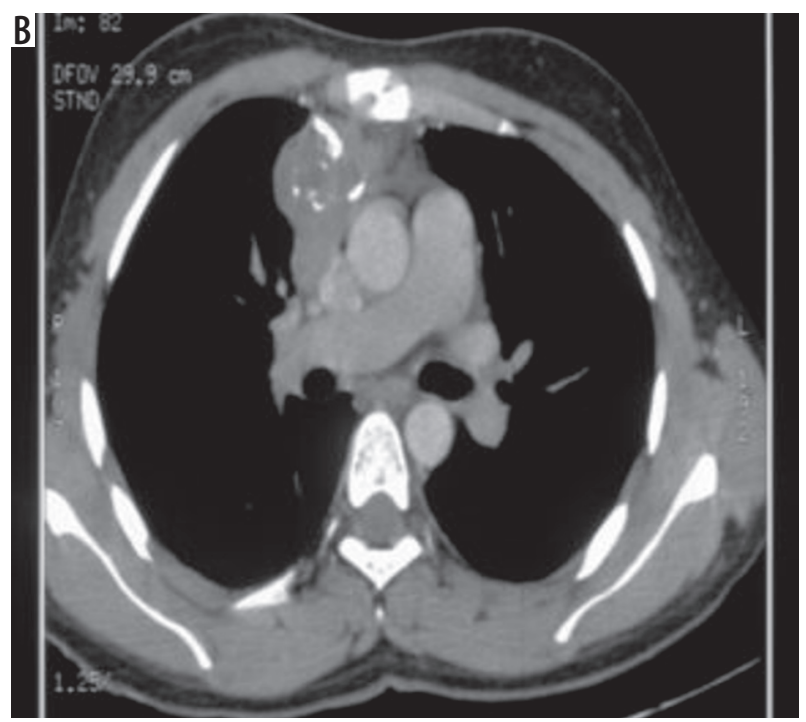

Figure 2. A) Mediastinal window - axial non-contrast images showing heterogeneous lobulated mass lesion with necrotic area and egg-shell calcification in prevascular space, which is isodense to muscle. B) Post-contrast axial images show heterogeneous enhancement of the mass lesion, which was seen abutting the major mediastinal vessels. C) Sections show a tumour composed of lobules separated by fibrous septa. Tumour is made up of mixture of epithelial cells and lymphoid cells. The epithelial cells have vesicular oval to round nuclei. Significant atypia or mitotic activity is not seen. The features are suggestive of thymoma type B2

peripheral rim enhancement $(n=13)$, intense enhancement $(n=3)$, and non-enhancing masses $(n=2)$. In the study the majority were solid masses, constituting $54 \%$ ( $n=27)$ of the cases, followed by solid and cystic masses in $22 \%(n=11)$ of the cases. In the study, $11 \%(n=7)$ of the mediastinum mass showed internal calcification. Necrosis was present in $45 \%$ of cases $(n=27)$.

Vascular invasion was the most common pattern, followed by chest wall invasion (Table 3). The most common lymph nodal group involved was subcarinal, which constituted $10 \%(n=6)$. Metastasis excluding to lymph nodal metastasis was present in $13 \%$ of cases $(n=8)$ (Table 4$)$.

\section{Discussion}

In this study dyspnoea was the most commonly presenting symptom, which was in concordance with Baram and Tayeb [2] in a study of 85 patients, in which they found that dyspnoea alone was the main presenting symptom in $33(38.82 \%)$ cases. In the present study of 60 cases, 21 benign and 39 malignant cases correlated with histopathological examination (Table 5). CT diagnosis did not 

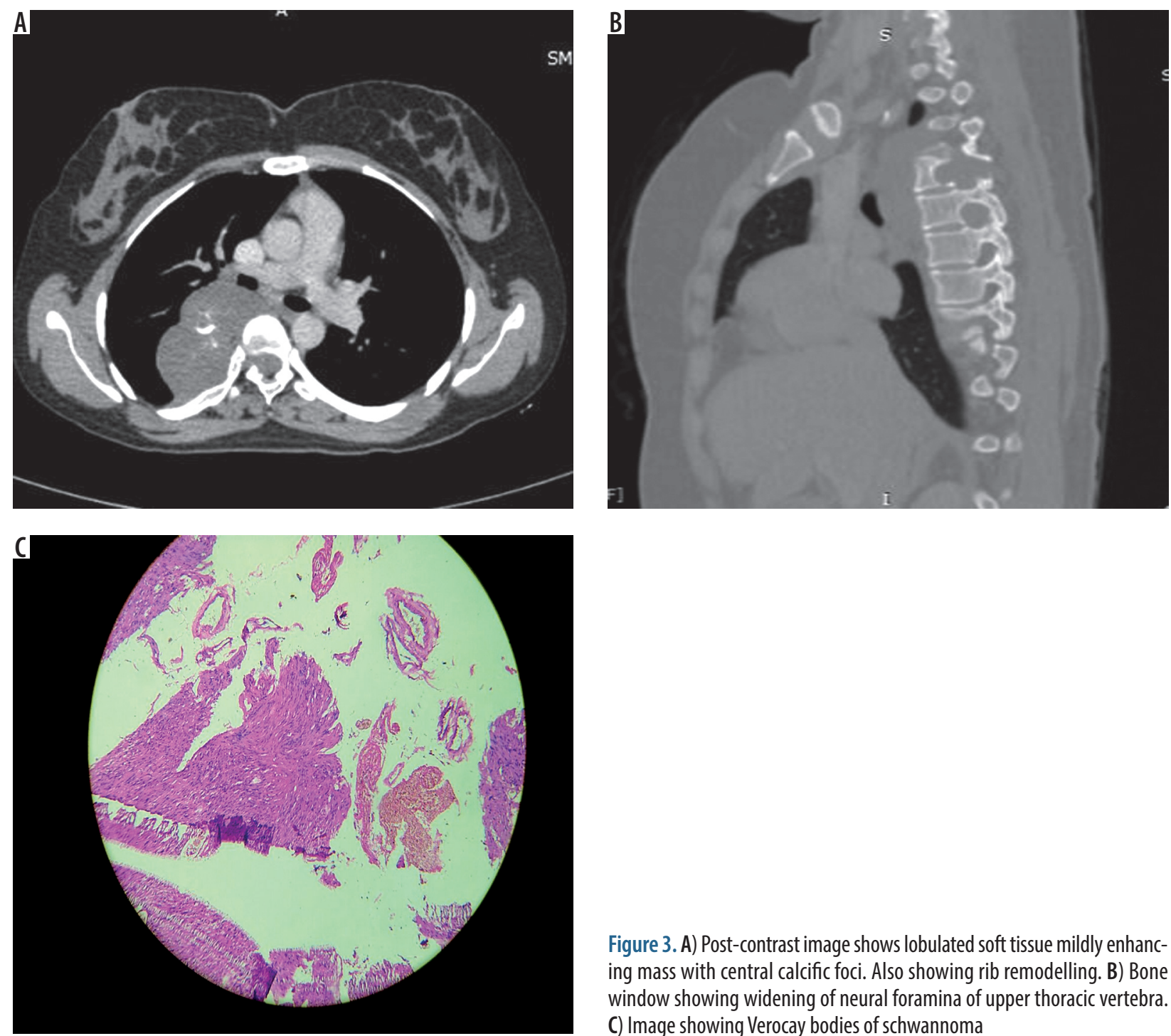

Figure 3. A) Post-contrast image shows lobulated soft tissue mildly enhancing mass with central calcific foci. Also showing rib remodelling. B) Bone window showing widening of neural foramina of upper thoracic vertebra. C) Image showing Verocay bodies of schwannoma

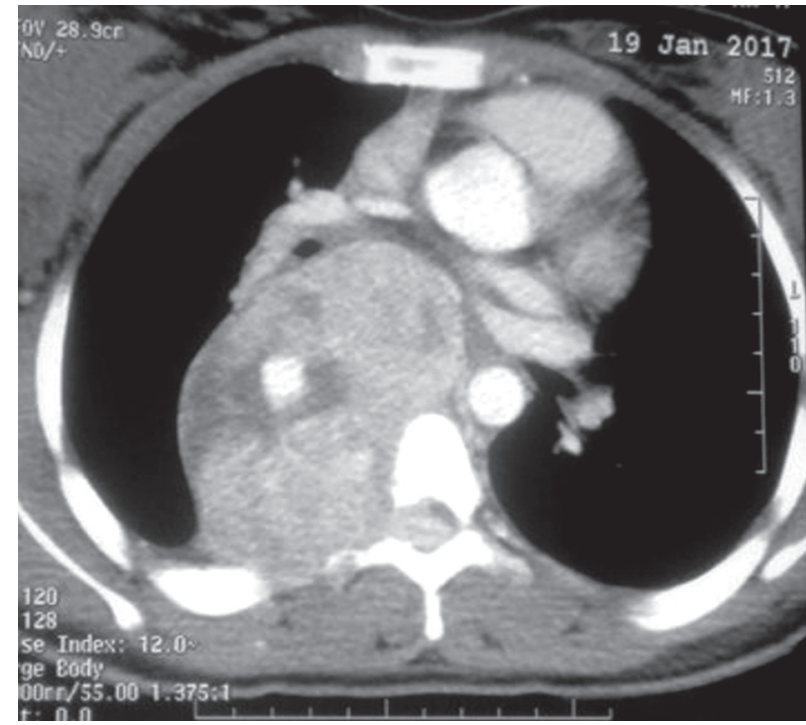

Figure 4. Post-contrast computed tomography showing intensely enhancing soft tissue mass lesion in paravertebral space. Lesion also shows aneurysmal dilatation of vessel inside mass. On histopathology it correlated well and was diagnosed as paraganglioma correlate with histopathological diagnosis in five cases (Tables 6 and 7). In this study the sensitivity of MDCT was $94 \%$ and specificity was $90 \%$, with a positive predictive value of $94 \%$, negative predictive value of $90 \%$, and diagnostic accuracy of $93 \%$ (Table 8), which is comparable with $\mathrm{Pu}$ lasani et al. [3], who found diagnostic accuracy of MDCT in the evaluation of mediastinal mass lesions to be $92 \%$. Mediastinal masses are more prevalent in the age group of 41-50 years, which is in concordance with work done by Dixit et al. [4]. In their study the most common age group was $30-50$ years. Out of 60 cases in the present study $65 \%$ were neoplastic and 35\% were non-neoplastic, which is comparable with Dixit et al. [4], who found that of 139 cases, 93 cases were neoplastic in nature (67\%). This finding was contrary to the study by Kaur et al. [5] in which benign pathology was much more common. Metastasis was the overall leading diagnosis in this study, and this is comparable with the study done by Adler et al. [6] in which they showed an appreciable proportion of metastatic lesions in the mediastinum. Among benign lesions tubercular infection was the most common in the present study at $11 \%$ 
Table 2. Multidetector computed tomography attenuation patterns of mediastinal masses

\begin{tabular}{|c|c|c|c|c|c|c|}
\hline \multirow[t]{3}{*}{ Attenuation } & \multicolumn{4}{|c|}{ Final diagnosis } & \multirow{2}{*}{\multicolumn{2}{|c|}{ Total }} \\
\hline & \multicolumn{2}{|c|}{ Benign } & \multicolumn{2}{|c|}{ Malignant } & & \\
\hline & No. & $\%$ & No. & $\%$ & No. & $\%$ \\
\hline Heterogeneous soft tissue & 8 & 38.10 & 21 & 53.85 & 29 & 48.33 \\
\hline Homogenous soft tissue & 4 & 19.05 & 11 & 28.21 & 15 & 25.00 \\
\hline Isodense soft tissue & 1 & 4.76 & 3 & 7.69 & 4 & 6.67 \\
\hline Homogenous soft tissue/Fat & 0 & 0.00 & 1 & 2.56 & 1 & 1.67 \\
\hline Isodense soft tissue/Fat & 1 & 4.76 & 0 & 0.00 & 1 & 1.67 \\
\hline Hyperdense soft tissue & 3 & 14.29 & 0 & 0.00 & 3 & 5.00 \\
\hline Hypodense soft tissue & 1 & 4.76 & 3 & 7.69 & 4 & 6.67 \\
\hline Fluid & 3 & 14.29 & 0 & 0.00 & 3 & 5.00 \\
\hline Total & 21 & 100.00 & 39 & 100.00 & 60 & 100.00 \\
\hline
\end{tabular}

Table 3. Invasion characteristic of different mediastinal masses

\begin{tabular}{|c|c|c|c|c|c|c|}
\hline \multirow{3}{*}{ Invasion } & \multicolumn{4}{|c|}{ Final diagnosis } & \multirow{2}{*}{\multicolumn{2}{|c|}{ Total }} \\
\hline & \multicolumn{2}{|c|}{ Benign } & \multicolumn{2}{|c|}{ Malignant } & & \\
\hline & No. & $\%$ & No. & $\%$ & No. & $\%$ \\
\hline None & 20 & 90.48 & 27 & 69.23 & 47 & 78.33 \\
\hline Yes & 1 & 9.52 & 12 & 30.77 & 13 & 21.67 \\
\hline Total & 21 & 100.00 & 39 & 100.00 & 60 & 100.00 \\
\hline
\end{tabular}

Table 4. Percentage and frequency of mediastinal masses showing solid organ metastasis

\begin{tabular}{|l|c|c|c|c|c|c|}
\multirow{2}{*}{ Mets to solid organs } & \multicolumn{4}{|c|}{ Final diagnosis } & \multicolumn{3}{c}{ Total } \\
\cline { 2 - 8 } & \multicolumn{2}{|c|}{ Benign } & \multicolumn{2}{c|}{ Malignant } & No. & $\%$ \\
\cline { 2 - 8 } & No. & $\%$ & 31 & 82.05 & 52 & 86.67 \\
\hline None & 21 & 100.00 & 8 & 17.95 & 8 & 13.33 \\
\hline Yes & 0 & 0.00 & 39 & 100.00 & 60 & 100.00 \\
\hline Total & 21 & 100.00 & & & & \\
\hline
\end{tabular}

$(n=7)$. In present study anterior/prevascular space followed by visceral compartment was most common location; however, Dubashi et al. [7] concluded that the majority of tumours were seen in the anterior mediastinum, followed by the posterior mediastinum; this result was partly favouring our study and partly against it. This was because we followed ITMIG classification, which includes oesophageal lesions in to the middle mediastinum. Thymoma was the leading radiological diagnosis in prevascular space, which was comparable with Araki et al. [8]. According to them, thymoma was the most common diagnosis in anterior mediastinum, and this finding in our study was contrary to Aroor et al. [9], who found lymphoma to be more common. Neurogenic tumour is the leading diagnosis in posterior mediastinum, being slight lower than in the study by Liu et al. [10], who found neurogenic tumour in $16.6 \%(n=68)$ of cases, while in our case it was $10 \%$, probably due to the small sample size in the present study.
The masses commonly found in multiple compartments were predominantly the lymph nodal masses, which was similar to the results of Kaur et al. [5]. Unusual mediastinal lesions in the present study were atypical primitive neuroectodermal tumour, plasmacytoma, and hydatid cyst. In our study vascular invasion was the most common finding constituting $18 \%(n=11)$ - aortic invasion was the most common at $8 \%(n=5)$ followed by superior vena cava invasion at $5 \%(n=3)$. Main pulmonary artery was involved in two cases; one of them turned out to be benign, i.e. tuberculoma. Heterogenous attenuation $p$ value $<0.05$, i.e. 0.036 , invasion $p$ value 0.23 , and metastasis $p$ value 0.42 were the only statistically significant MDCT characteristics that were more common with malignant lesions, and they were helpful in differentiating them from benign entities. Necrosis, compartment localisation, enhancement, and presence or absence of calcification was statistically insignificant. 
Table 5. Multidetector computed tomography findings and final histopathological diagnosis

\begin{tabular}{|c|c|c|c|c|}
\hline Mediastinal lesions & Final diagnoses & Percentage & CT diagnosis & Percentage \\
\hline Thymoma & 6 & 10 & 6 & 10 \\
\hline Thymic hyperplasia & 2 & 3.33 & 2 & 3.33 \\
\hline Thymic carcinoma & 1 & 1.66 & 1 & 1.66 \\
\hline Thyroid goitre & 2 & 3.33 & 2 & 3.33 \\
\hline Hodgkin & 3 & 5 & 3 & 5 \\
\hline Non-Hodgkin & 2 & 3.33 & 2 & 3.33 \\
\hline Tubercular lymph node & 5 & 8.33 & 5 & 8.33 \\
\hline Reactive lymph node & 1 & 1.66 & 0 & 0 \\
\hline Germ cell tumour & 2 & 3.33 & 2 & 3.33 \\
\hline Metastatic lymph node & 8 & 13.3 & 6 & 10 \\
\hline Lymphangioma & 1 & 1.66 & 1 & 1.66 \\
\hline Acute myeloid leukaemia & 1 & 1.66 & 1 & 1.66 \\
\hline Neurogenic tumour & 6 & 10 & 6 & 10 \\
\hline $\begin{array}{l}\text { Atypical primitive } \\
\text { neuroectodermal tumor }\end{array}$ & 1 & 1.66 & 0 & 0 \\
\hline Oesophageal carcinoma & 7 & 11.6 & 7 & 11.6 \\
\hline Potts spine & 3 & 5 & 3 & 5 \\
\hline Pyogenic spondylodiscitis & 1 & 1.66 & 1 & 1.66 \\
\hline Hydatid cyst & 1 & 1.66 & 1 & 1.66 \\
\hline Plasmacytoma & 1 & 1.66 & 1 & 1.66 \\
\hline Oesphageal duplication cyst & 1 & 1.66 & 1 & 1.66 \\
\hline Bronchogenic cyst & 3 & 5 & 3 & 5 \\
\hline Mediastinal abscess & 1 & 1.66 & 1 & 1.66 \\
\hline Parathyroid adenoma & 1 & 1.66 & 0 & 0 \\
\hline Total & 60 & 100 & 55 & \\
\hline
\end{tabular}

Table 6. Histopathological correlation

\begin{tabular}{|l|c|c|c|}
\hline Computed tomography diagnosis & Histopathologic diagnoses & HPE benign & HPE malignant \\
\hline Reactive lymph node & Parathyroid adenoma & - & + \\
\hline Tubercular lymphadenopathy & Metastatic lymph node & - & + \\
\hline Nonseminomatous germ cell tumour & Atypical primitive neuroectodermal tumor & - & + \\
\hline Sarcoidosis & Reactive lymph node & + & - \\
\hline Primary lung carcinoma with metastatic lymph node & Tuberculoma & + & - \\
\hline
\end{tabular}

Table 7. Frequency and percentage of benign and malignant mediastinal masses based on multidetector computed tomography and histopathological findings

\begin{tabular}{|l|c|c|c|c|c|c|}
\multirow{2}{*}{ CT diagnosis } & \multicolumn{4}{c|}{ Final Diagnosis } & \multicolumn{3}{c|}{ Total } \\
\cline { 2 - 8 } & \multicolumn{2}{|c|}{ Benign } & \multicolumn{2}{c|}{ Malignant } & No. & $\%$ \\
\cline { 2 - 8 } & No. & $\%$ & No. & $\%$ & 21 & 35.00 \\
\hline Benign & 19 & 90.48 & 2 & 5.13 & 39 & 65.00 \\
\hline Malignant & 2 & 9.52 & 37 & 94.87 & 60 & 100.00 \\
\hline Total & 21 & 100.00 & 39 & 100.00 & 60 \\
\hline
\end{tabular}


Table 8. Diagnostic efficiency of multidetector computed tomography

\begin{tabular}{|l|c|c|}
\hline Diagnostic efficiency & No. & $\%$ \\
\hline Sensitivity & $37 / 39$ & 94.87 \\
\hline Specificity & $19 / 21$ & 90.48 \\
\hline PPV & $37 / 39$ & 94.87 \\
\hline NPV & $19 / 21$ & 90.48 \\
\hline Accuracy & $56 / 60$ & 93.33 \\
\hline
\end{tabular}

MDCT has been specifically shown to have a high accuracy for characterising the size, location, and adjacent organ involvement in the evaluation of mediastinal masses $[11,12]$ as well as affecting clinical management in $65 \%$ of cases and adding additional diagnostic information in $82 \%$ [11-13]. MDCT features of mediastinal masses are as follows:

\section{Thymoma}

Thymomas accounts for $20 \%$ of adult mediastinal masses $[14,15]$, which are rarely also found in children. Homogenous soft tissue in prevascular space with absence of lymphadenopathy is a supportive finding. Necrosis and internal calcification occurs in $30 \%$ of thymomas. Thymomas also show mild enhancement.

\section{Thymic hyperplasia}

On MDCT, thymic hyperplasia is characterised by diffuse symmetric homogeneous enlargement of thymus having areas of fat and soft tissue attenuation and smooth contour. If asymmetric enlargement presents, then thymoma is likely to be the diagnosis. MRI chemical-shift imaging is the modality of choice to differentiate between thymic hyperplasia and carcinoma because of its ability to detect microscopic fat present in thymic hyperplasia. In lymphofollicular hyperplasia, on histopathology, the medulla of the gland shows hyperplastic lymphoid germinal matrix in the centre. This entity is commonly noted in patients with myasthenia gravis and various other autoimmune disorders.

\section{Germ cell tumours}

Teratomas - these are the most common germ cell tumour. Most are benign - also known as mature teratomas. Malignant teratomas are also called immature type, accounting for $14 \%$ of cases. Both benign and malignant teratomas are characterised by presence of fluid, fat, and calcific foci. The presence of enhancing soft tissue suggests a diagnosis of malignant teratoma [16-19].

Seminoma - these lesions presents as large, homogeneous, lobulated, soft tissue mass with mild enhancement. Calcification is typically absent, and invasion is not a feature of seminoma. The diagnostic accuracy of CT alone to differentiate between non-teratomatous germ cell tumour and other malignant prevascular mediastinal masses is $35 \%[20]$.

\section{Thyroid masses}

These present as hyper-attenuating masses because of the high iodine content; intense early and prolonged enhancement is also a key feature. Necrosis may be present. Calcifications are commonly seen in masses of thyroid origin.

\section{Ectopic parathyroid adenoma}

These are soft tissue hypoattenuating lesion with mild enhancement, mimicking an enlarged lymph node, typically showing greater contrast enhancement. For a symptomatic ectopic parathyroid gland in prevascular space, thoracoscopic or open resection is indicated.

\section{Mediastinal lymphoma}

Hodgkin disease accounts for 50-70\% of mediastinal lymphomas. These lesions have a bimodal age distribution in young adults and in patients older than 50 years. These present as a homogeneous soft-tissue mass in the prevascular space with surface lobulation and mild-to-moderate contrast enhancement with absence of vascular involvement [20]. Cystic changes and necrosis are occasional. Hodgkin lymphoma and non-Hodgkin lymphoma typically appear similar to each other; although NHL causes more vascular involvement, pleural and pericardial effusion is common in all type of lymphomas. Reactive lymph nodes may be mistaken for lymphomas; however, reactive lymph nodes do not coalesce into large masses, which is common with lymphomas.

\section{Tubercular lymphadenopathy}

On CT, the enlarged lymph nodes show a highly characteristic "rim sign" caused by a low-density centre surrounded by an enhancing peripheral rim [21], but this sign is not pathognomonic for tubercular lymphadenopathy because metastases (from head/neck/testicular cancer), infection with atypical mycobacteria, and histoplasmosis may also show similar rim enhancement. Lymphoma may also present with rim enhancement [22]. So, pulmonary pa- 
renchymal abnormalities must be sought before making a diagnosis of tubercular lymphadenopathy.

\section{Bronchogenic cyst}

Present as round to ovoid low attenuation lesions which may have soft tissue density if they have been complicated by haemorrhages or have a proteinaceous component which may show enhancement.

\section{Oesophageal carcinoma}

Characterised by eccentric or circumferential thickening of the oesophageal wall, with loss of perioesophageal fat plane. Invasion may also be present. May lead to abnormal air fluid level inside the oesophagus.

\section{Oesophageal duplication cyst}

Presents as well-defined homogenous fluid attenuation lesion usually having a thicker wall.

\section{Lymphangiomas}

These lesions are more common in prevascular space on CT. These are characterised by smooth homogeneous lobulated lesions of water attenuation containing cysts of variable size. Contrast enhancement and calcification are the least common. They may show occasional chylous pleural effusion. Mediastinal lymphangiomas produce symptoms when causing compression of surrounding structures. Infection and haemorrhage may complicate the lesion. The treatment of choice is total excision [23].

\section{Mediastinal abscess}

When the collection contains air and has a thick rim enhancing wall, an abscess should be suspected. Pleural effusion, mediastinal air-fluid levels, or even a soft-tissue density mass may also be evident on CT [23].

\section{Neurogenic tumours}

Most incidental neurogenic tumours in older age are benign. On CT, they appear as well-defined, paravertebral masses with punctate or curvilinear calcifications. They may also show necrosis. Intraspinal extension is best demonstrated on MRI.

\section{Schwannomas}

Peripheral nerve tumours on CT appear as round, well-defined lesions with smooth margins and are isodense or hypodense to the adjacent chest wall musculature. They have a variable enhancement pattern after contrast material. Cystic changes and haemorrhage are more common in schwannomas than in neurofibromas, frequently affecting patients from 20 to 30 years old [24].

\section{Mediastinal paraganglioma}

One to two percent of extra-adrenal paragangliomas occur in the thorax [24]. These are intensely enhancing homogenous soft tissue lesion of posterior mediastinum, and they may have necrosis.

\section{Sternal plasmacytoma}

Presents similarly to metastasis on CT, as lytic lesions, soft-tissue masses, with a multilobulated appearance. Intrinsic calcifications are absent, and expansile lesions also occur.

\section{Paravertebral abscess}

Pyogenic infection is suspected when a patient presents with back pain and fever and on imaging ill-defined soft tissue, unorganised fluid, and/or a loculated collection are present in the paravertebral. Infection secondary to tuberculosis involves the posterior elements, presents with a prevertebral and paravertebral soft-tissue component out of proportion to the degree of osseous destruction; narrowing of disk space enables differentiation of tuberculous from pyogenic infection. The presence of calcification in the absence of new bone formation or sclerosis strongly suggests the diagnosis of tubercular spondylodiscitis $[25,26]$.

\section{Mediastinal PNET}

PNETs (primitive neuroectodermal tumours) are extremely rare in mediastinum. Zhang et al. [27] concluded that EWS/PNETs in the anterior and middle mediastinum appear as ill-defined, heterogeneous masses that are not distinguishable from other, more common causes of mediastinal masses, based on their CT features.

\section{Mediastinal hydatid}

Hydatid cyst are uncommon in mediastinum. Hydatid cyst shows fluid attenuation on CT. The presence of membranes and presence of hydatid in another location, especially liver, is enough to suggest diagnosis of hydatid cyst. Mediastinal hydatid may show calcification [28]. In a series of 14 cases of mediastinal hydatid studied by Zidi et al. [29] anterior mediastinum was the most common location.

\section{Conclusions}

Mediastinal mass lesion can often be diagnosed with great confidence based on location and imaging features alone. In other scenarios, a diagnosis may be suggested when radiologic features are combined with specific clinical in- 
formation. Benign and malignant masses can be differentiated with great accuracy. MDCT sometimes fails to differentiate between solid and cystic lesion as in the case of complicated bronchogenic cyst, so in these circumstances MRI is helpful. Differentiation between rim-enhancing lymph nodes is challenging - to categorise as metastatic or tubercular. Invasion, heterogenous attenuation, and metastasis are key parameters to differentiate between benign and malignant mediastinal lesions. MDCT is a cost effective noninvasive tool, which must be used with other modalities, e.g. PET and MRI in diagnosis and management of mediastinal mass lesion whenever conditions demand.

\section{Conflict of interest}

The authors report that there is no conflict of interest.

\section{References}

1. Carter BW, Benveniste MF, Madan R, et al. ITMIG Classification of Mediastinal Compartments and Multidisciplinary Approach to Mediastinal Masses. Radiographics 2017; 37: 413-436.

2. Baram A, Tayeb ZA. Mediastinal Masses: Retrospective Single Center Based Study. J Cancer Sci Ther 2016; 8: 252-256.

3. Pulasani K, Narayanaswamy I, Ramprakash HV. Evaluation of Mediastinal Mass Lesions Using Multi-detector Row Computed Tomography and Correlation with Histopathological Diagnosis. Int J Sci Stud 2015; 3: 156-163.

4. Dixit R, Shah NS, Goyal M, et al. Diagnostic evaluation of mediastinal lesions: Analysis of 144 cases. Lung India 2017; 34: 341-348.

5. Kaur H, Tiwari P, Dugg P, et al. Computed tomographic evaluation of mediastinal masses/lesions with contrast enhancement and correlation with pathological diagnosis - a study of 120 cases. J Biomed Graph Comput 2014; 4: 28-35.

6. Adler OB, Rosenberger A, Peleg H. Fine-needle aspiration biopsy of mediastinal masses: Evaluation of 136 experiences. AJR Am J Roentgenol 1983; 140: 893-896.

7. Dubashi B, Cyriac S, Tenali SG. Clinicopathological analysis and outcome of primary mediastinal malignancies. A report of 91cases from a single institute. Ann Thorac Med 2009; 4: 140-142.

8. Araki T, Nishino M, Gao W, et al. Anterior mediastinal masses in the Framingham Heart Study: Prevalence and CT image characteristics. Eur J Radiol Open 2015; 2: 26-31.

9. Aroor AR, Prakasha SR, Seshadri S, et al. A study of clinical characteristicsof mediastinal mass. J Clin Diagn Res 2014; 8: 77-80.

10. Liu T, Al-Kzayer LF, Xie X, et al. Mediastinal lesions across the age spectrum: a clinicopathological comparison between pediatric and adult patients. Oncotarget 2017; 8: 59845-59853.

11. ee EY. Evaluation of non-vascular mediastinal masses in infants and children: an evidence-based practical approach. Pediatr Radiol 2009; 39 Suppl 2: S184-S190.

12. aeber GM, Shriver CD, Albus RA, et al. The use ofcomputed tomography in the evaluation of mediastinal masses. J Thorac Cardiovasc Surg 1986; 91: 662-666.

13. Siegel MJ, Sagel SS, Reed K. The value of computed tomography in the diagnosis and management of pediatric mediastinal abnormalities. Radiology 1982; 142: 149-155.

14. Ranganath SH, Lee EY, Restrepo R, et al. Mediastinal masses in children. Am J Roentgenol 2012; 198: W197-W216.
15. Nasseri F, Eftekhari F. Clinical and radiologic review of the normal and abnormal thymus: pearls and pitfalls. Radiographics 2010; 30: 413-428.

16. Merten DF. Diagnostic imaging of mediastinal masses in children. Am J Roentgenol 1992; 158: 825-832.

17. Meza MP, Benson M, Slovis TL. Imaging of mediastinal masses in children. Radiol Clin North Am 1993; 31: 583-604.

18. Moeller KH, Rosado-de-Christenson ML, Templeton PA. Mediastinal mature teratoma: imaging features. AJR Am J Roentgenol 1997; 169: 985-990

19. Rosado-de-Christenson ML, Templeton PA, Moran CA. From the archives of the AFIP. Mediastinal germ cell tumors: radiologic and pathologic correlation. Radiographics 1992; 12: 1013-1030.

20. 20. Tomiyama N, Honda O, Tsubamoto M, et al. Anterior mediastinal tumors: diagnostic accuracy of CT and MRI. Eur J Radiol 2009; 69: 280-288.

21. Kim WS, Moon WK, Kim IO, et al. Pulmonary tuberculosis in children: Evaluation with CT. AJR Am J Roentgenol 1997; 168: 10051009.

22. Van Dyck P, Vanhoenacker FM, Van den Brande P, De Schepper AM. Imaging of pulmonary tuberculosis. Eur Radiol 2003; 13: 17711785 .

23. Shahrzad M, Lee TS, Silva M, et al. Anterior Mediastinal Masses. AJR Am J Roentgenol 2014; 203: W128-W138.

24. Nakazono T, White CS, Yamasaki F, et al. MRI findings of mediastinal neurogenic tumors. AJR Am J Roentgenol 2011; 197 : W643-W652.

25. De Backer AI, Mortelé KJ, Vanschoubroeck IJ, et al. Tuberculosis of the spine: CT and MR imaging features. JBR-BTR 2005; 88: 92-97.

26. Engin G, Acunaş B, Acunaş G, Tunaci M. Imaging of extrapulmonary tuberculosis. Radiographics 2000; 20: 4713.

27. Zhang WD, Zhao LL, Huang XB, et al. Computed tomography imaging of anterior and middle mediastinal Ewing sarcoma/primitive neuroectodermal tumors. J Thorac Imaging 2010; 25: 168-172.

28. von Sinner WN, Linjawi T, al Watban JA. Mediastinal hydatid disease: report of three cases. J Can Assoc Radiol 1990; 41: 79-82.

29. Zidi A, Zannad-Hantous S, Mestiri I, et al. Hydatid cyst of the mediastinum: 14 case reports. J Radiol 2006; 87 (12 Pt 1): 1869-1874. 